MECHANISMS OF DISEASE

\section{Kinases out of control - brake line cut!}

Activating mutations of receptor tyrosine kinases (RTKs) are responsible for various human cancers and other diseases. Reporting in Molecular Cell, Chen and colleagues show that a triad of residues makes up a molecular brake that inhibits RTK activation. Pathogenic mutations that result in increased RTK activity disengage this brake, either directly or indirectly.

Previous patient screenings had uncovered several pathogenic mutations in the RTK fibroblast growth-factor receptor-2 (FGFR2). Measuring the intrinsic kinase activity of several pathogenic FGFR2 mutants, Chen and colleagues found that several of these mutations conferred higher rates of activation (A) loop autophosphorylation and peptide substrate phosphorylation.

To find out why this might be, the authors determined the structures of the unphosphorylated and autophosphorylated forms of the kinase domain of wild-type FGFR2 (FGFR2K). Crucially, upon autophosphorylation the N-terminal lobe of the kinase domain rotates inwards, towards the C-terminal lobe. Furthermore, a triad of residues (E565, N549 and K641) form a structural network of hydrogen bonds in the kinase hinge region of unphosphorylated FGFR2K, but not in autophosphorylated FGFR2K. The authors propose that this network of hydrogen bonds functions as a molecular brake that inhibits $\mathrm{N}$-terminal rotation and thereby keeps the kinase in an autoinhibited conformation.

To confirm this finding, the authors determined the structures of seven unphosphorylated pathogenic FGFR2K mutants, of which five had a mutation in residue E565, N549 or K641. Indeed, mutation of these residues disengaged the network directly. Moreover, the other two mutations disengaged the network indirectly through long-range allostery that involves the A-loop and $\alpha \mathrm{C}$ helix regulatory regions.

Importantly, the three residues that constitute the brake are conserved in all FGFRs and in other RTKs - including plateletderived growth-factor receptors and vascular endothelial growth-factor receptors - and retrospective analysis of crystal structures revealed that the same inhibitory hydrogen-bond network exists in many RTKs. Thus, this molecular brake represents a major conserved mechanism of RTK regulation. Pathogenic mutations disengage this molecular brake and make it easier for RTKs to adopt their activated conformation. Whether a broken molecular brake can be repaired to slow disease pathogenesis remains to be determined.

Asher Mullard

ORIGINAL RESEARCH PAPER Chen, H. et al. A molecular brake in the kinase hinge region regulates the activity of receptor tyrosine kinases. Mol. Cell 27 , 717-730(2007)
DOI:

$10.1038 / \mathrm{nrm} 2280$

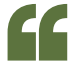

... a triad of

residues makes

up a molecular

brake that

inhibits RTK

activation
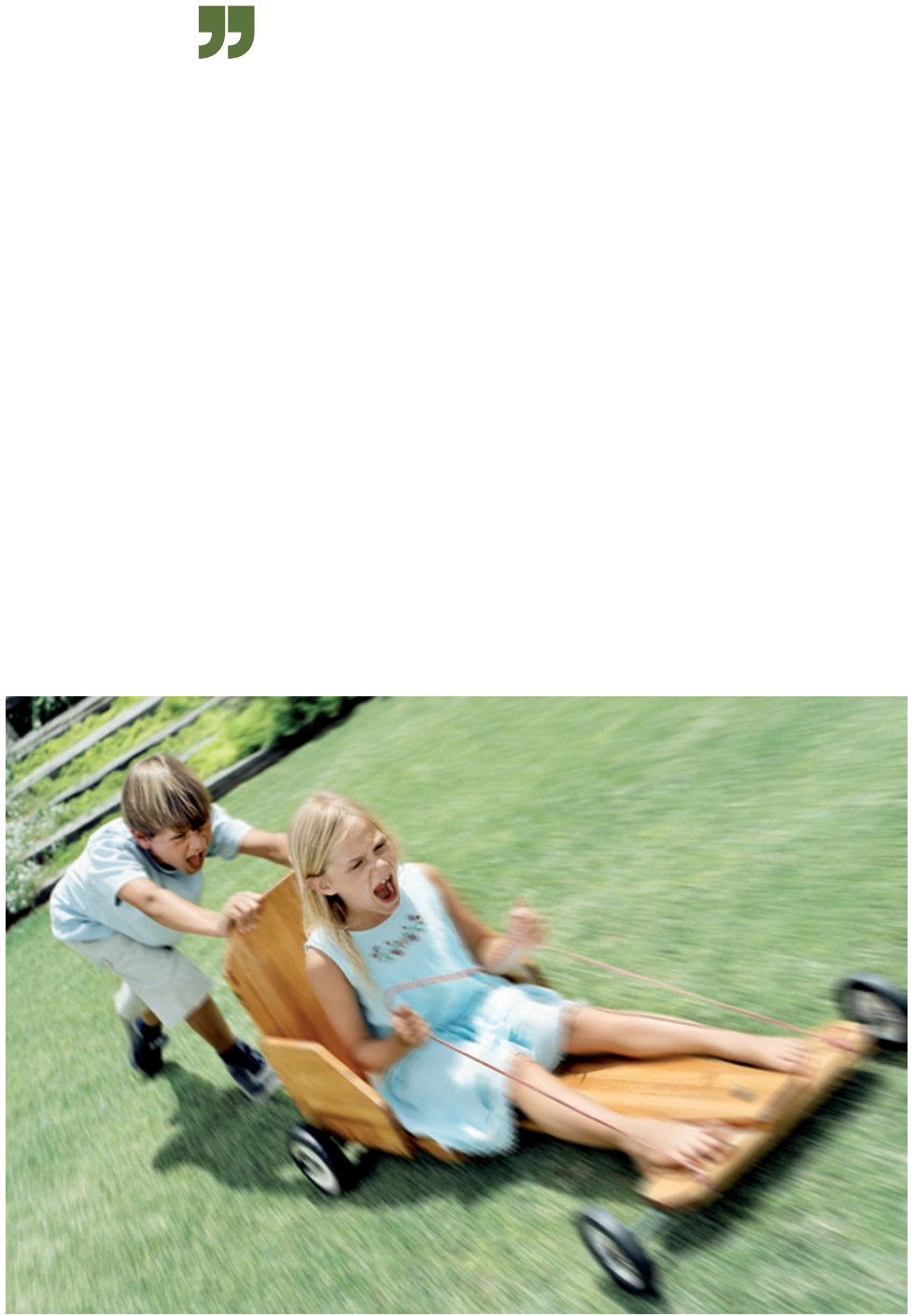